\title{
Design of Eccentric Training System Based on Multiple-Input Single-Output Wiener Nonlinear Model
}

\author{
Chunbo Liu
}

\begin{abstract}
Objectives: The efficacy of eccentric cycling has been proved in research, clinical, and sport training activities. However, several constraints make it difficult to use commercially available eccentric cycle ergometers. This study will stimulate the application of eccentric cycling as an exercise modality. Study Design: University research laboratory. Methods: In this study, the multipleinput single-output (MISO) Wiener nonlinear model was applied to the design of the eccentric training system. Particle Swarm Optimization (PSO) algorithm was then applied to identify the parameters of the MISO Wiener nonlinear model. The computer software embedded with MISO model was programmed for not only controlling and monitoring the running status of the eccentric training system, but also collecting data in a flexible and convenient manner. Results: The correlation coefficient of 0.9985 and root mean square error of 3.3264 between the collected power value and the estimated power value from the model shows the good performance of the model. Conclusion: The proposed method for the design of the eccentric training system using MISO Wiener nonlinear model provides a new way for eccentric research and training.
\end{abstract}

Keywords: eccentric cycling, ergometer, Wiener model, modeling

Eccentric cycling was first introduced in $1952,{ }^{1}$ where 2 interlinked bicycles were used with one person pedaling forward (ie, concentric) and the other resisting the backward movements (ie, eccentric) imposed on the bicycle. Since then, researchers have been intrigued by the observation that eccentric cycling (cycle ergometry adapted to impose active muscle lengthening) can be performed at a considerably lower metabolic cost compared with traditional concentric cycling (cycle ergometry used to impose active muscle shortening). ${ }^{2}$

Chronic eccentric cycling (6-12 wk) has been demonstrated as a potent stimulus for improving locomotor muscle function in a variety of populations. These include patients with Parkinson's disease, cancer survivors, total knee arthroplasty patients, individuals with anterior cruciate ligament injuries, elderly individuals, young healthy individuals, and athletes. The efficacy of eccentric cycling has attracted researchers to adopt eccentric cycling as a model for exercise interventions.

Although the efficacy of eccentric cycling has been proved, some potential barriers prevented the eccentric cycling becoming popular, such as limited number of commercially available eccentric cycle ergometers. To overcome such difficulty, the components used to build an eccentric cycle ergometer have been briefly described..$^{3-5}$ However, these early ergometers were constructed using mostly custom parts that resulted in rather complex and costly designs.

With the advancements in technology, engineering solution based on mathematical model is making it possible to construct a modern eccentric cycle ergometer, which can be used in research, clinical, and sport training settings in a flexible and convenient manner. This study proposed 1 engineering solution based on multiple-input single-output (MISO) Wiener nonlinear model, and the solution provides a new way for the design of eccentric training system.

The author is with the School of Sport Science, Exercise and Health, Faculty of Science, The University of Western Australia, Crawley, WA, Australia. Liu (chunbo.liu@uwa.edu.au) is corresponding author.

\section{Methods}

\section{MISO Wiener Nonlinear Model}

The mathematical model was applied to indirectly get accurate eccentric power that people worked on the eccentric trainer. When the electric motor drives the trainer during the eccentric experiment, the whole system power $P_{(\text {Whole })}$ can be divided into 2 parts $P_{(\text {Part } 1)}$ and $P_{(\text {Part } 2)}: P_{(\text {Part } 1)}$ was the power for driving whole trainer including the pedal without load and $P_{(\operatorname{Par} 2)}$ was the power for conquering the eccentric power that people worked on the eccentric trainer. In other words, eccentric power was negative, but the value of electricity power $P_{(\text {Part } 2)}$ was equal to the eccentric power that people worked on the eccentric trainer. So $P_{(\text {Whole })}$ can be described as the following:

$$
P_{(\text {Whole })}=P_{(\text {Part } 1)}+P_{(\text {Part } 2)} .
$$

$P_{(\text {Whole })}$ can be gained through the high precision power meter and $P_{(\text {Part } 1)}$ can be gained by mathematical modeling. In detail, first, the power data of the eccentric trainer without loading were collected during different speeds; second, the data were used to construct the power model of the eccentric trainer during different speeds without loading. So $P_{(\text {Part } 2)}$ can be calculated by the following equation:

$$
P_{(\text {Part } 2)}=P_{(\text {Whole })}-P_{(\text {Part } 1)} \text {. }
$$

For applying MISO Wiener nonlinear model to gain $P_{(\text {Part1 })}$, some notations were introduced first here. The superscript $T$ denotes the matrix transpose; $I$ stands for an identity matrix of appropriate sizes; the norm of a matrix $X$ is defined by $\|X\|^{2}=\operatorname{tr}\left[X X^{T}\right]$; and $z^{-1}$ is a unit backward shift operator, that is, $z^{-1} y(k)=y(k-1)$. Consider an MISO Wiener nonlinear system shown in Figure 1:

$$
\begin{gathered}
x(k)=\sum_{i=1}^{r} G_{i}(p, z) u_{i}(k), \\
y(k)=f(x(k)),
\end{gathered}
$$




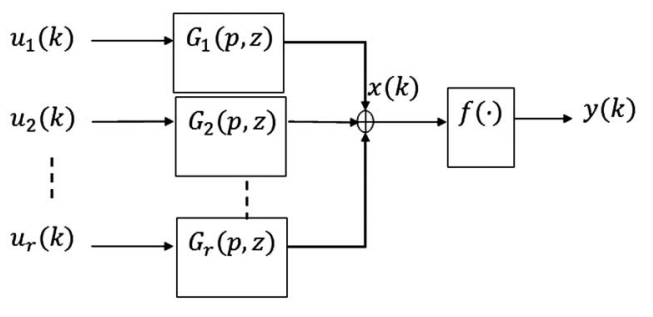

Figure 1 - Multiple-input single-output Wiener nonlinear model.

where $\left\{u_{i}(k), i=1,2, \ldots, r\right\}$ are the system inputs; $x(k)$ is the output of the linear part; $y(k)$ is the system output and the linear part of the Wiener system is described by $r$ finite impulse response models with the $p$ orders' transfer function, defined by

$$
G_{i}(p, z)=a_{i 1}+a_{i 2} z^{-1}+\ldots+a_{i p} z^{-p+1} .
$$

The nonlinear part $f(\cdot)$ of the system is represented by a linear combination of $n_{\gamma}$ nonlinear basis functions:

$$
f(x(k))=\gamma_{1} f_{1}(x(k))+\gamma_{2} f_{2}(x(k))+\ldots+\gamma_{n_{\gamma}} f_{n_{\gamma}}(x(k)) .
$$

In this paper, the special case was considered when the static nonlinearity $f(\cdot)$ was assumed invertible so that the internal variable associated with the output $y(k)$ can be reconstructed as:

$$
\begin{aligned}
x(k) & =f^{-1}(y(k)) \\
& =c_{1} g_{1}(y(k))+c_{2} g_{2}(y(k))+\ldots+c_{n_{c}} g_{n_{c}}(y(k)),
\end{aligned}
$$

where $f^{-1}(\cdot)$ denotes the inverse of $f(\cdot), g_{i}(\cdot)\left(i=1,2, \cdots, n_{c}\right)$ are the known basis functions, and $c i\left(i=1,2, \cdots, n_{c}\right)$ are the unknown parameters. Without loss of generality, we set the first coefficient of the function $f^{-1}(\cdot)$ to be 1 , that is, $c_{1}=1$ for identifiability. Substituting Equation (3) into Equation (7) gives:

$$
\begin{aligned}
g_{1}(y(k))= & \sum_{i=1}^{r} G_{i}(p, z) u_{i}(k)-c_{2} g_{2}(y(k))-c_{3} g_{3}(y(k))-\ldots \\
& -c_{n_{c}} g_{n_{c}}(y(k)) .
\end{aligned}
$$

Note that the nonlinearity considered here covers cubic spline nonlinearity, ${ }^{6}$ piecewise linear functions with discontinuities, ${ }^{7}$ and polynomial nonlinearities. ${ }^{8}$ According to Ding et al, ${ }^{9}$ the polynomial nonlinearity was introduced as the nonlinear part of the Wiener system, and Equation (8) was transformed into:

$$
y(k)=\sum_{i=1}^{r} G_{i}(p, z) u_{i}(k)-c_{2} y^{2}(k)-c_{3} y^{3}(k)-\ldots-c_{n_{c}} y^{n_{c}}(k) \text {. }
$$

Define the information vectors and the parameter vectors

$$
\begin{gathered}
\varphi_{s}(k)=\left[u_{1}(k), u_{1}(k-1), \ldots, u_{1}(k-p+1),\right. \\
u_{2}(k), u_{2}(k-1), \ldots, u_{2}(k-p+1), \\
\left.u_{r}(k), u_{r}(k-1), \ldots, u_{r}(k-p+1)\right]^{T} \in \mathbb{R}^{r p} \\
\varphi_{c}(k)=\left[-y^{2}(k),-y^{3}(k), \ldots,-y^{n_{c}}(k)\right]^{T} \in \mathbb{R}^{n_{c}-1} \\
\varphi(k)=\left[\begin{array}{c}
\varphi_{s}(k) \\
\varphi_{c}(k)
\end{array}\right] \in \mathbb{R}^{r p+n_{c}-1} \\
\theta_{s}=\left[a_{11}, a_{12}, \ldots, a_{1 p}, a_{21}, a_{22}, \ldots, a_{2 p}, a_{r 1}, a_{r 2}, \ldots, a_{r p}\right]^{T} \in \mathbb{R}^{r p} \\
\theta_{c}=\left[c_{2}, c_{3}, \ldots, c_{n_{c}}\right]^{T} \in \mathbb{R}^{n_{c}-1}
\end{gathered}
$$

$$
\theta=\left[\begin{array}{c}
\theta_{s} \\
\theta_{c}
\end{array}\right] \in \mathbb{R}^{r p+n_{c}-1}
$$

From Equation (9), the following identification model was obtained:

$$
y(k)=\phi_{s}^{T}(k) \theta_{s}+\phi_{c}^{T}(k) \theta_{c}=\phi^{T}(k) \theta .
$$

$P_{(\text {Part } 1)}$, the model was:

$$
y(k)=a_{11} u_{1}(k)+a_{12} u_{1}(k-1)+c_{2} y^{2}(k)+c_{3} y^{3}(k) .
$$

A particle swarm optimization (PSO) algorithm was used to estimate the unknown parameter vector $\theta$ for the MISO Wiener nonlinear system.

\section{PSO Algorithm}

Particle swarm optimization algorithm is an evolutionary computation technique. It was first proposed by Kennedy and Eberhart in 1995. ${ }^{10}$ The basic idea mainly refers to the characteristics of swarm intelligence. PSO can be simply formulated as follows: Each potential solution to the object function is assumed as a particle, searching the optimal value via cooperation at certain velocity. It is an iterative algorithm, and each particle alters its velocity and position dynamically by the following equations:

$$
\begin{gathered}
v_{i j}^{n+1}=w v_{i j}^{n}+c_{1} r_{1}\left(p_{i j}^{n}-x_{i j}^{n}\right)+c_{2} r_{2}\left(p_{g i}^{n}-x_{i j}^{n}\right), \\
x_{i j}^{n+1}=x_{i j}^{n}+v_{i j}^{n+1}, \\
w=w_{\min }+\left(w_{\max }-w_{\min }\right) \\
\times\left(\text { interation }_{\max }-\text { interation }_{i}\right) / \text { interation }_{\max },
\end{gathered}
$$

where $v_{i j}$ is the $j$ th dimension of the $i$ th particle's velocity, and it is usually confined to the closed interval of $\left[v_{\min }, v_{\max }\right]$ to prevent the explosion of the particles. The notation of $x_{i j}, p_{i j}$, and $p_{g i}$ is similar to that of $v_{i j}$. Coefficients $r_{1}$ and $r_{2}$ are 2 randomly generated values within the range of $[0,1]$ for the $d$ th dimension. $c_{1}$ and $c_{2}$ are 2 acceleration parameters commonly set to 2.0 or adaptively controlled according to the evolutionary states. Factor $w$ is the inertial weight, which plays the role of controlling the impact of the previous velocity of a particle on the current one so as to balance between the global search (large inertial weight) and the local search (small inertial weight). $w_{\max }$ and $w_{\min }$ represent the maximum and minimum inertial weights and are always set to 0.9 and 0.4 , respectively. Interation max $_{\text {ax }}$ is the maximum number of allowed iterations, whereas interation ${ }_{i}$ represents the current iteration.

\section{Structure of Eccentric Training System}

The mechanical parts of the eccentric trainer including ergometer frame, electric motor, frequency drive, and industrial chain, which were similar to the mechanical parts as previously described. ${ }^{2}$ The eccentric trainer was driven by an electric motor that was controlled by a frequency drive. As the frequency drive can give different electrical signal frequency to control the speed of the electric motor, the speed of the pedal connected to the electric motor by the industrial chain can also be controlled at the set value. The high precision (Accuracy $\pm 0.5 \%$ ) power meter UPM209 Energy Analyser (Algodue Elettronica, Fontaneto d'Agogna, Italy) was used for monitoring the whole power of the training system. The upper computer was used for collecting data and controlling the eccentric trainer automatically. 
Microsoft Visual Studio VB.net (Microsoft, Redmond, WA) was applied for designing the software part. The RS485 serial port by Modbus-RTU/ASCII protocol was used for the communications among the computer, UPM209 Energy Analyser, and frequency drive.

\section{Results}

Based on the collected power value of the eccentric training system, the parameters of the MISO Wiener nonlinear model for the eccentric training system were finally identified by the PSO algorithm. The parameters versus generations were shown in

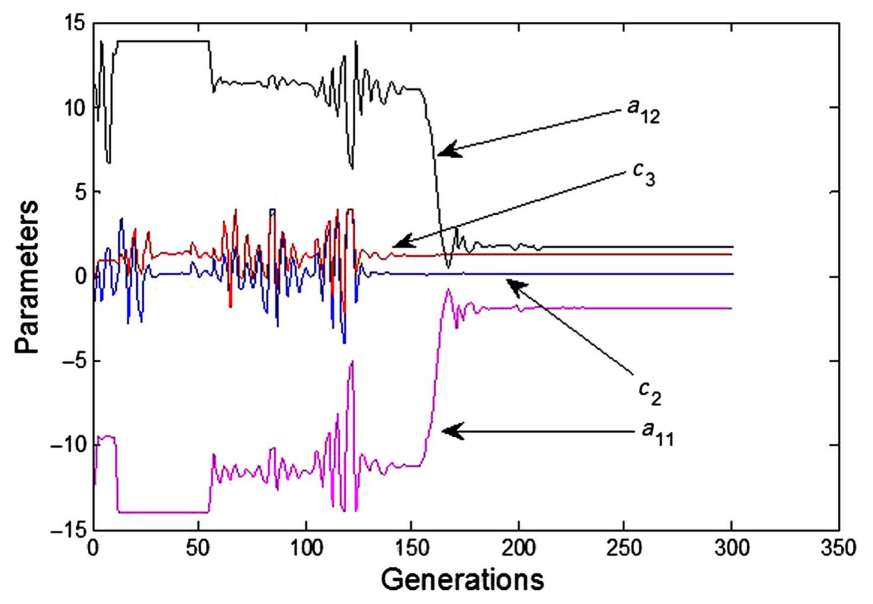

Figure 2 - Parameters of MISO Wiener nonlinear model versus generations. As marked by arrows: the black line represents $a_{12}$, red line represents $c_{3}$, blue line represents $c_{2}$, and pink line represents $a_{11}$ for the parameters of MISO Wiener nonlinear model as listed in Equation (11). MISO indicates multiple-input single-output.
Figure 2, and the root mean square errors versus generations finally dropped to 3.7136 . The mathematical model was constructed as follows:

$$
\begin{aligned}
y(k)= & -1.8517 u_{1}(k)+1.6926 u_{1}(k-1)+0.1304 y^{2}(k) \\
& +1.2835 y^{3}(k) .
\end{aligned}
$$

The correlation coefficient and root mean square error between the 2 different types of value was .9985 and 3.3264, respectively, which proved the performance of the model.

The software on the eccentric trainer running in the computer was shown in Figure 3. If the real-time eccentric power value falls beyond the threshold safety value, the eccentric trainer will stop itself automatically for safety protection. The real-time power and speed at the same time were also shown, and the elapsing training time was gained from the clock timer. The eccentric training speed was set through pressing the button "SPEED UP," "SPEED DOWN," or being input manually. The training data were saved through pressing the button "Save Data" for the future data analysis.

\section{Discussion}

The use of eccentric cycling is impeded due to the barriers, such as limited number of commercially available eccentric cycle ergometers, manufacture constraints, and substantial cost. Although previous studies described the components used to build an eccentric cycle ergometer, these ergometers were constructed using mostly custom parts that resulted in rather complex and costly designs. ${ }^{3-5}$ Electric motors have also been used to drive the backward rotations of the cranks. In addition, a modern eccentric cycle ergometer has been constructed, for which the Schoberer Rad Messtechnik (SRM) power meter (Jülich, Germany) was used to quantify the eccentric power value; however, the SRM was difficult to calibrate, which resulted in the unreliability of the collected data.

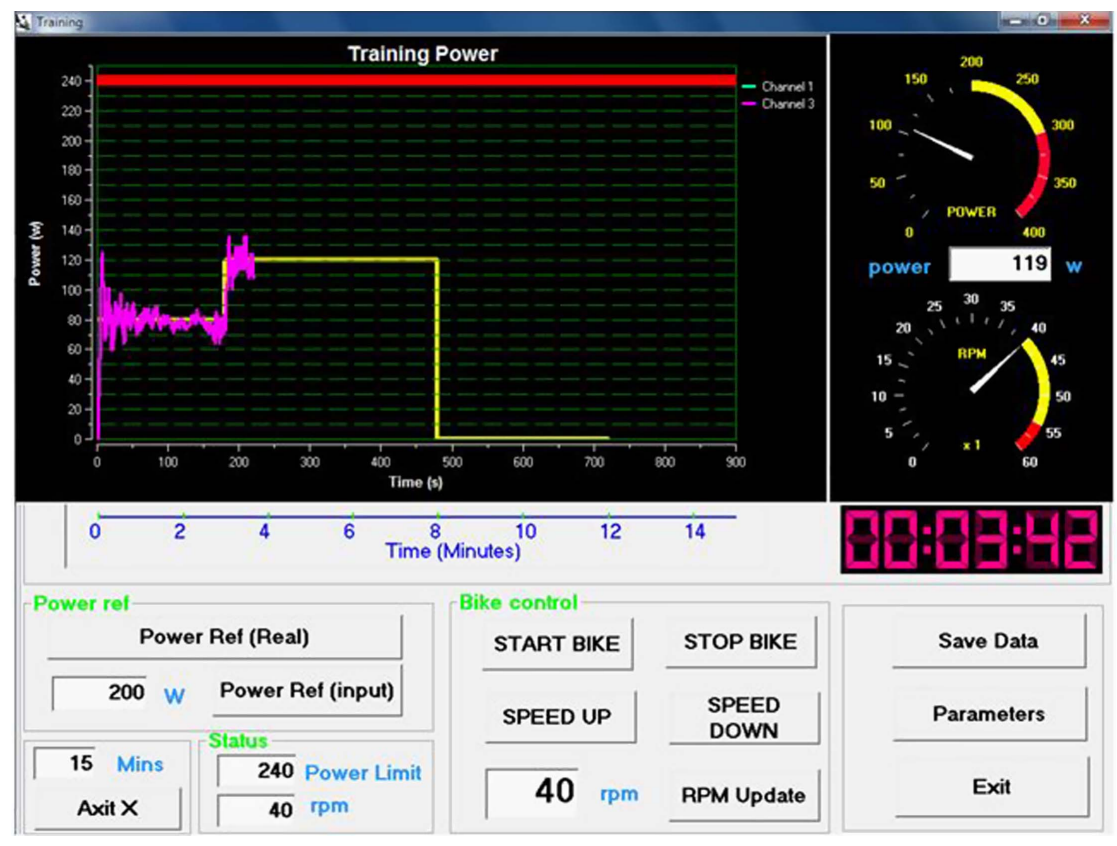

Figure 3 - Operation panel of eccentric training system. The collected eccentric power value is indicated in the pink line (rapidly changing one at the lower area), the yellow line (relatively stable one at the lower area) represents the reference power line for tester during the eccentric training, and the safety threshold line is shown in red (stable one at the higher area). 
Furthermore, SRM was mainly designed for cycling purpose and the SRM operation panel cannot match the eccentric study purpose.

This study proposed an alternative way for the design of eccentric training system by the application of MISO Wiener nonlinear model. To ensure the accuracy of the system, following the Equation (2), the collected whole system power $P_{(\text {Whole })}$ and the estimated power $P_{(\text {Part } 1)}$ for driving the whole trainer including the pedal without loading must be of high accuracy. In terms of $P_{(\text {Whole })}$, the accuracy of the UPM209 Energy Analyser was within 0.5\%; for $P_{(\text {Part } 1)}$, the mathematical model captured $99.85 \%$ variance of the eccentric trainer power, and the accuracy of $P_{(\text {Part } 2)}$ was within $1 \%$.

There are 3 considerations to ensure the safety during the running of the eccentric training system. First, the safety switch is provided to the tester, and the system is functional only when the switch is pressed down by the tester. Second, the safety warning line can be set by the tester such as the red line (stable one at the higher area) in Figure 3, and if the training power exceeds the safety warning line, the eccentric training system will stop itself automatically and generates the warning signal to draw the attention of tester. Third, the emergency stop button is set in front of the tester, and the whole eccentric training system will stop immediately if the button is pressed.

In addition, the proposed method in this study allows reversing the mounting of the entire system so that the thread would prevent loosening. The issue of loosing is 1 common problem during the application of power meters because the threading on the bottom bracket and the pedals is only oriented to prevent loosening during concentric cycling, not during eccentric cycling.

In summary, the proposed method for the design of eccentric training system using MISO Wiener model provides an alternative way for the eccentric research and training. Due to the shortcomings of the current commercial eccentric cycle ergometers, this study will stimulate the application of the eccentric cycling as an exercise modality.

\section{References}

1. Abbott BC, Bigland B, Ritchie J. The physiological cost of negative work. J Physiol. 1952;117(3):380-390. PubMed ID: 14946742 doi: 10.1113/jphysiol.1952.sp004755

2. Elmer SJ, Martin JC. Construction of an isokinetic eccentric cycle ergometer for research and training. J Appl Biomech. 2013;29(4): 490-495. PubMed ID: 22923268 doi:10.1123/jab.29.4.490

3. Bigland-Ritchie B, Graichen H, Woods JJ. A variable-speed motorized bicycle ergometer for positive and negative work exercise. J Appl Physiol. 1973;35(5):739-740. PubMed ID: 4770358 10.1152/jappl. 1973.35.5.739

4. Knuttgen HG, Patton JF, Vogel JA. An ergometer for concentric and eccentric muscular exercise. J Appl Physiol Respir Environ Exerc Physiol. 1982;53(3):784-788. PubMed ID: 7130003

5. Petersen FB. A bicycle ergometer for investigating the effect of eccentric exercise with arms and legs. Int Z Angew Physiol. 1969; 27(2):133-137. PubMed ID: 5788675

6. Dempsey EJ, Westwick DT. Identification of Hammerstein models with cubic spline nonlinearities. IEEE Trans Biomed Eng. 2004; 51(2):237-245. PubMed ID: 14765696 doi:10.1109/TBME.2003. 820384

7. Voros J. Parameter identification of wiener systems with multisegment piecewise-linear nonlinearities. Syst Control Lett. 2007;56(2): 99-105. doi:10.1016/j.sysconle.2006.08.001

8. Ding F, Shi Y, Chen TW. Gradient-based identification methods for Hammerstein nonlinear ARMAX models. Nonlinear Dynam. 2006; 45(1-2):31-43. doi:10.1007/s11071-005-1850-z

9. Ding F, Chen TW, Iwai Z. Adaptive digital control of Hammerstein nonlinear systems with limited output sampling. Siam J Control Optim. 2007;45(6):2257-2276. doi:10.1137/05062620X

10. Kennedy J, Eberhart R. Particle swarm optimization. Proc IEEE Int Conf Neural Netw. 1995;4(1-6):1942-1948. doi:10.1109/ICNN. 1995.488968 